\title{
Proton Pump Inhibitor Therapy Associated with Lower Glycosylated Hemoglobin Levels in Type 2 Diabetes
}

\author{
Michael A. Crouch, MD, MSPH, Ivan N. Mefford, MD, PhD, and \\ Ekpedeme U. Wade, MD
}

Introduction: Oral hypoglycemic medications sometimes do not control type 2 diabetes well. Proton pump inhibitors (PPIs) as adjunctive therapy might improve diabetes control through increasing serum gastrin and fasting insulin levels.

Methods: Electronic medical records in a family medicine residency program office practice were reviewed for 73 individuals with type 2 diabetes (not taking insulin), for whom PPIs were prescribed. Values for glycosylated hemoglobin (HbA1c) for periods of time when a PPI had been prescribed were compared with HbA1c levels for periods of time with no record of PPI prescribing or over-the-counter PPI use.

Results: The mean HbA1c for patients not taking insulin was 7.11 during periods with recorded prescribing or over-the-counter use of PPIs, compared with 7.70 during periods without recorded PPI therapy $(P=$ .001). Mean HbA1c for metformin monotherapy was not significantly different (6.81 with PPI vs. 7.10 without PPI; $n=16 ; P=.25)$. Mean HbA1c was significantly different for combination therapy that included metformin and/or sulfonylurea and/or giltazone (7.26 vs. $7.80 ; n=27 ; P=.002)$.

Conclusion: The observed association between PPI therapy and lower HbA1c levels suggests that PPIs may be useful as adjunctive therapy for type 2 diabetes. (J Am Board Fam Med 2012;25:50-54.)

Keywords: Glycosylated Hemoglobin A, Hb A1c, Proton Pump Inhibitors, Type 2 Diabetes Mellitus

The prevalence of type 2 diabetes continues to rise; it now affects about 25 million people in the United States and 285 million people worldwide. ${ }^{1,2}$ Type 2 diabetes is characterized by insulin resistance and/or deficient pancreatic $\beta$-cell mass or production and secretion of insulin. ${ }^{3,4}$

Mefford and Wade have reviewed animal and human evidence showing that gastrin plays a sig-

This article was externally peer reviewed.

Submitted 12 July 2010; revised 28 June 2011; accepted 7 July 2011.

From the Memorial Family Medicine Residency Program, Sugar Land, Texas (MC); and Fort Bend Premier Care, Richmond, Texas (INM).

Funding: none.

Conflict of interest: none declared.

Corresponding author: Michael A. Crouch, 14023 Southwest Freeway, Sugar Land, TX 77478 (E-mail: michael.crouch@ memorialhermann.org).

\section{See Related Article on Page 5.}

nificant role in pancreatic function and glucose regulation. ${ }^{5-18}$ Proton pump inhibitors (PPIs), used to treat excess stomach acid, indigestion, gastroesophageal reflux disease, and gastric ulcers, can lead to consistently elevated serum gastrin levels. ${ }^{18-25}$ Data on the relationship between PPI therapy and diabetes control is scanty in the literature. Mefford and Wade's retrospective chart review found a significantly lower mean glycosylated hemoglobin (HbA1c) for patients with type 2 diabetes (not receiving insulin therapy) who previously had been prescribed a PPI. ${ }^{5}$

The purpose of this study was to see if the reported association between PPI therapy and lower HbA1c levels would be seen in patients with type 2 diabetes during time periods on PPI therapy versus time periods off PPI therapy.

\section{Methods}

Electronic medical records (Logician/Centricity) in the office practice of a community hospital-based 
family medicine residency program were searched for adult patients with an active diagnosis of type 2 diabetes mellitus, controlled or uncontrolled (International Classification of Diseases [ICD] codes 250.00 or 250.02 , respectively), with at least 2 documented office visits between September 4, 2007, and June 30, 2009. Charts were audited and data were abstracted for those who had at least one documented HbA1c result following a period of time during which there was documented prescribing or over-the-counter (OTC) use of a PPI and at least one HbA1c result following a period of time during which there was no documented prescribing or OTC use of a PPI.

Patient charts were excluded from auditing if there was documentation of insulin or sustained corticosteroid therapy during the time frame covered by the audit. At the time of the audit, the office practice contained medical records for approximately 2800 adults with a diagnosis of type 2 diabetes (controlled [ICD code 250.00] or uncontrolled [ICD code 250.02]), of whom almost 300 had PPIs on their medication lists. To check for possible interaction effect, the principal investigator abstracted and analyzed data regarding (1) documented HbA1c results, (2) diabetes medications on the medications list, (3) PPI medications on the medications list, and (4) sex, age, and ethnicity. The study protocol was approved by the University of Texas, Houston, Institutional Review Board.

\section{Data Analysis}

The Statistical Package for the Social Sciences (version 15.0, IBM, Chicago, IL) was used to compare the mean HbA1c levels of diabetic patients taking different diabetes medications alone and in combination, with and without accompanying PPI therapy. The 2 -sided $t$ test (significance level of $P<$ $.05)$ was used for the main analyses comparing mean HbA1c levels for patients receiving any hypoglycemic therapy or taking other classes and combinations of classes of diabetes medications with versus without concomitant PPI therapy. Analysis of covariance was used to check for interaction effects with age, sex, and ethnicity.

\section{Results}

A total of 71 diabetic patients had documented HbA1c values for periods of time during which PPIs had been and had not been prescribed or used in OTC form. All of these patients also were prescribed various oral hypoglycemic agents, but none had insulin prescribed during the time frame covered by the chart audit. The mean age was 62.6 years (range, $32-87$ years); 45 patients $(63.3 \%)$ were women and $26(36.7 \%)$ were men (Table 1$)$. Of the patient sample, 24 (33.8\%) were AfricanAmerican, 23 (32.4\%) were non-Hispanic white, 17 (23.9\%) were Hispanic, 5 (7\%) were Asian, and 2 (2.8\%) were unknown. These proportions are similar to the overall ethnic composition of this clinic practice.

The mean HbA1c for 71 patients not using exogenous insulin was 7.12 during periods with recorded prescribing or OTC use of PPI therapy compared with 7.71 during periods without recorded PPI prescribing or OTC use $(P=.001$; Figure 1).

Mean $\mathrm{HbA1c}$ for patients receiving metformin monotherapy $(\mathrm{n}=16)$ was not significantly different (HbA1c of 6.80 with PPI vs 7.10 without PPI; $P=.25$; Figure 2), but mean HbA1c was significantly different for patients receiving combination therapy that included metformin and/or sulfonylurea and/or giltazone therapy $(\mathrm{n}=27$; HbA1c of 7.26 with PPI vs 7.80 without PPI being prescribed or used OTC; $P=.002$; Figure 3 ). There was no significant interaction effect detected for age, sex, or ethnicity.

\section{Discussion}

These findings are partially consistent with those reported for the only previous study with a similar design. ${ }^{26}$ Mefford and Wade's retrospective

Table 1. Demographic Characteristics of Study Sample

\begin{tabular}{lc}
\hline Age (years) & \\
\hline Mean & 62.6 \\
Median & 62 \\
Range & $32-87$ \\
Sex (n [\%]) & \\
Female & $45(63.3)$ \\
Male & $26(36.7)$ \\
Ethnicity (n [\%]) & \\
African American & $24(33.8)$ \\
Asian & $5(7)$ \\
Non-Hispanic white & $23(32.4)$ \\
Hispanic & $17(23.9)$ \\
Unknown & $2(2.8)$ \\
\hline
\end{tabular}


Figure 1. Mean hemoglobin A1c with and without an active proton pump inhibitor (PPI) prescription.

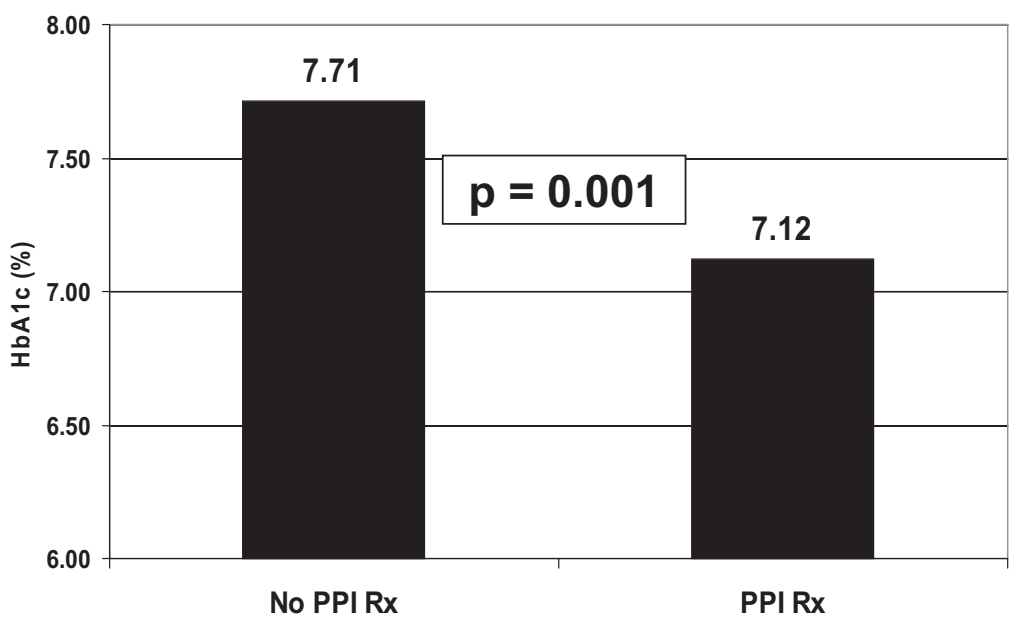

chart review for 347 individuals with type 2 diabetes who were not taking insulin therapy showed a mean HbA1c of 7.0 for patients who previously had been prescribed a PPI compared with 7.6 for patients who had not been prescribed a PPI $(P=.002) .{ }^{5}$ Mean HbA1c differences for patients taking specific hypoglycemic agents were significant for those receiving metformin monotherapy (mean HbA1c was $6.6 \%$ on PPI vs $7.3 \%$ not on PPI; $P<.05$ ) and sulfonylurea with or without metformin and/or thiazolidenedione (glitazone; mean HbA1c was $6.5 \%$ on PPI vs 7.9\% not on PPI; $P<.001) .^{5}$

Both chart audit studies showed that PPI therapy was associated with significantly lower levels of $\mathrm{HbA1c}$ in patients with type 2 diabetes who were taking oral hypoglycemic agents but not insulin. In this study, the association was seen only during times of active PPI therapy, whereas the other study suggested a lingering association for some months after PPI therapy was discontinued. In both studies, mean HbA1c levels were significantly lower for patients who had PPIs prescribed or used OTC along with combination hypoglycemic therapy consisting of metformin and/or glitazone and/or sulfonylurea. This study found a statistically insignificant difference in $\mathrm{HbA1c}$ for patients with prescribed or OTC PPI use along with metformin monotherapy in contrast to the significant difference reported for the other chart audit study. ${ }^{26}$

The findings of this study provide modest support for the proposition that PPIs may be a useful

Figure 2. Mean hemoglobin A1c with a prescription for metformin with and without a concomitant proton pump inhibitor (PPI).

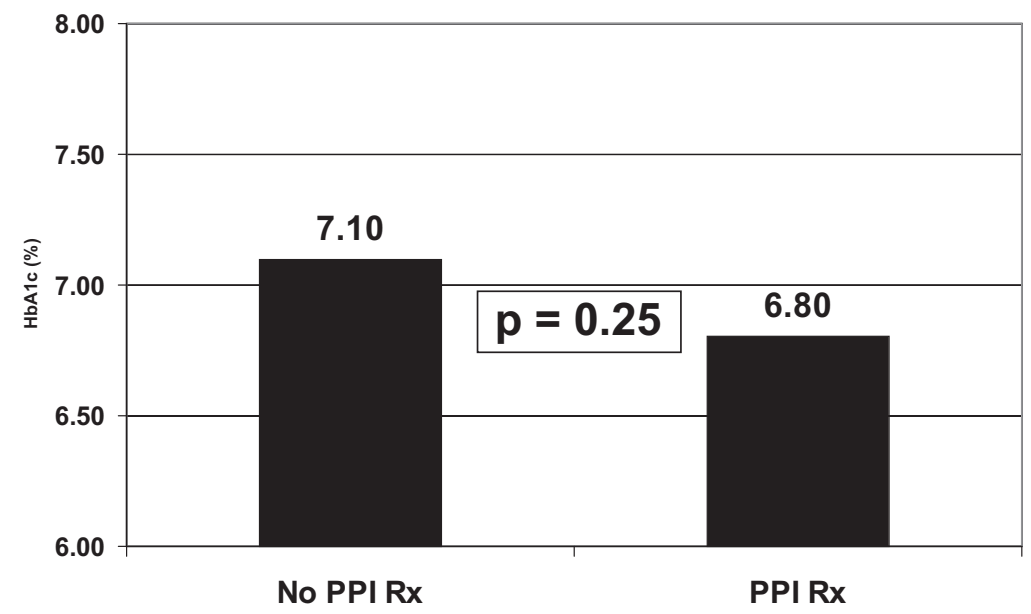


Figure 3. Mean hemoglobin A1c with sulfonylurea and/or glitazone and/or metformin with and without a prescription for a concomitant proton pump inhibitor (PPI).

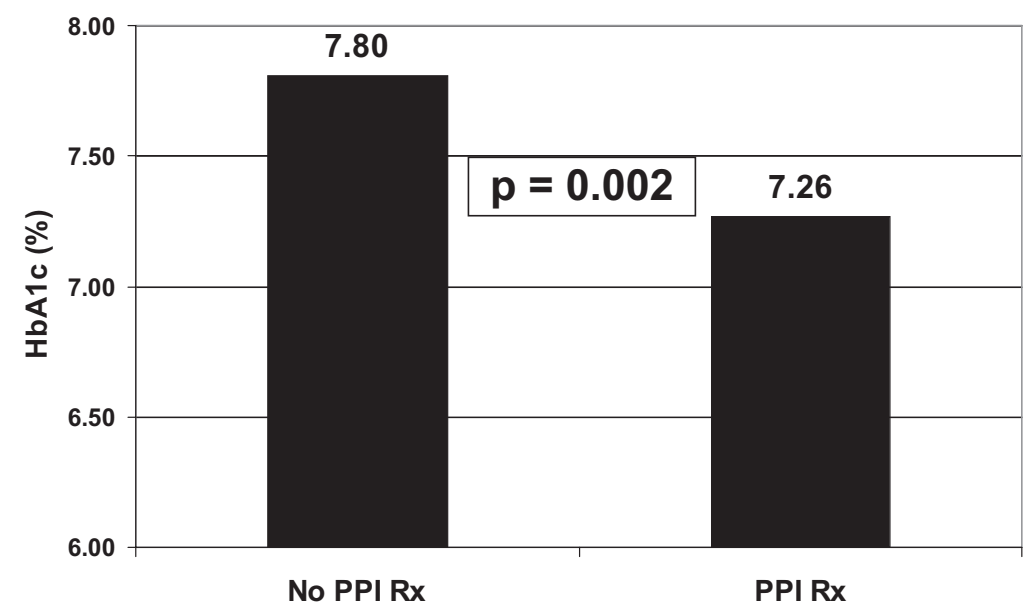

adjunctive therapy for type 2 diabetes. The postulated mechanism for this effect is PPI causing elevation of serum gastrin, enhancing pancreatic $\beta$-cell function, and stimulating increased insulin secretion.

The major limitation of this retrospective chart audit study is that it demonstrates only a statistical association between prescribed PPI therapy and lower HbA1c levels. This association does not necessarily result from a causal relationship. Another limitation of this study is the lack of medication adherence information for both diabetes medications and PPIs. Thus the data analysis essentially is based on documented known intention to treat. Given the high incidence of gastroesophageal reflux (the main indication for PPI therapy), it is probable that some patients were taking over-thecounter PPIs or PPIs prescribed by physicians outside this practice that were not documented in the medical records that were audited.

The described association merits further investigation with clinical trials measuring gastrin, insulin, and HbA1c levels for patients with type 2 diabetes (not taking insulin), off and on adjunctive PPI therapy, which the authors (INM, EUW) are now conducting.

\section{Conclusion}

PPIs may be a useful adjunctive therapy for type 2 diabetes. Clinical trials are warranted to further investigate this possibility.

\section{References}

1. Cowie CC, Rust KF, Ford ES, et al. Full accounting of diabetes and pre-diabetes in the U.S. population in 1988-1994 and 2005-2006. Diabetes Care 2009; 32:287-94.

2. Shaw J, Sicree R, Zimmet P. Global estimates of the prevalence of diabetes for 2010 and 2030. Diabetes Res Clin Pract 2009;87:4-14.

3. Mahler RJ, Adler ML. Type 2 diabetes mellitus: update on diagnosis, pathophysiology, and treatment. J Clin Endo Metab 1999;84:1165-71.

4. Bell DSH. Current status of diabetes treatment. South Med J 2002;95:24-9.

5. Mefford IN, Wade EU. Proton pump inhibitors as a treatment method for type II diabetes. Medl Hypotheses 2009;73:29-32.

6. Suarez-Pinzon WL, Yan Y, Rabinovitch A. Combination therapy with gastrin and glucagon-like peptide-1 (GLP-1) restores pancreatic insulin content and corrects hyperglycemia after diabetes onset in NOD mice. Can J Diabetes 2004;28:257.

7. Rooman I, Lardon J, Bouwens L. Gastrin stimulates B-cell neogenesis and increases islet mass from transdifferentiated but not from normal exocrine pancreas. Diabetes 2002;51:686-90.

8. Cowey SL, Quast M, Belalcazar LM, et al. Abdominal obesity, insulin resistance and colon carcinogenesis are in creased in mutant mice lacking gastrin gene expression. Cancer 2005;103:2643-53.

9. Schubert ML, Makhlouf GM. Neural, hormonal and paracrine regulation of gastrin and acid secretion. Yale J Biol Med 1992;65:553-60.

10. Parkinson J. A case of pernicious anaemia terminating in acute diabetes. Lancet 1910;II:416-8.

11. Rehfeld JF. Disturbed islet-cell function related to endogenous gastrin release. J Clin Invest 1976;58: $41-9$. 
12. Rehfeld JF, Stadil F, Baden H, Fischermann K. The enteral insulin-stimulation after Whipple's operation. Diabetologia 1975;11:207-10.

13. Bryant JG, Smith JV. Zollinger-Ellison syndrome with pancreatic islet cell hyperplasia. Calif Med 1965;102:49-52.

14. Meier JJ, Butler AE, Galasso R, Rizza RA, Butler $\mathrm{PC}$. Increased islet beta cell replication adjacent to intrapancreatic gastrinomas in humans. Diabetologia 2006;49:2689-96.

15. Yoshihisa U., Susumu I, Yasuyuki M, et al. Effect of serum gastrin concentration on insulin resistance. Digestion Absorption 2006;28:42-5.

16. Creutzfeldt $W$. The consequences of hypergastrinemia. Yale J Biol Med 1994;67:709-23.

17. Gurden G, Boyacioglu S, Gul C, et al. Impact of Helicobacter pylori infection on serum gastrin in haemodialysis patients. Nephrol Dial Transplant 1999;14:2688-91.

18. Peach HG, Barnett NE. Helicobacter pylori infection and fasting plasma glucose concentration. J Clin Path 2001;54:466-9.

19. Hu Y-M, Mei Q, Xu X-H, Hu X-P, Hu N-Z, Xu J-M. Pharmacodynamic and kinetic effect of rabeprazole on serum gastrin level in relation to CYP2C19 polymorphism in Chinese Hans. World J Gastroenterol 2006;12:4750-3.
20. Ligumsky M, Lysy J, Siguencia G, Friedlander Y. Effect of long-term, continuous versus alternate-day omeprazole therapy on serum gastrin in patients treated for reflux esophagitis. J Clin Gastroenterol 2001;33:32-5.

21. Cadiot G, Vissuzaine C, Pospai D, Ruszniewski P, Potet F, Mignon M. Effect of prolonged treatment with proton pump inhibitors on serum gastrin levels and the fundus mucosa. Gastroenterol Clin Biol 1995;19:811-7.

22. Koop H, Klein M, Arnold R. Serum gastrin levels during long-term omeprazole treatment. Aliment Pharmacol Therap 1990;4:131-8.

23. Vanderhoff BT, Tahboub RM. Proton pump inhibitors: an update. Am Fam Physician 2002;66:273-80.

24. Sanders SW, Tolman KG, Greski PA, Jennings DE, Hoyos PA, Page JG. The effects of lansoprazole, a new $\mathrm{H}+, \mathrm{K}(+)$-ATPase inhibitor, on gastric $\mathrm{pH}$ and serum gastrin. Aliment Pharmacol Ther 1992;6: 359-72.

25. Banerjee S, Ardill JES, Beattie AD, McColl KEL. Effect of omeprazole and feeding on plasma gastrin in patients with achlorhydria. Aliment Pharmacol Ther 1995;9:507-12.

26. Suzaki F, Suzuki R, Sugiyama M. An increase of serum insulin by induced hypergastrinemia with proton pump inhibitor in type 2 diabetes. Prog Med 2004;24:661-4. 